\title{
Traduire
}

Une eutre perspective sur $r$ tatadciction

Revue française de la traduction

$240 \mid 2019$

Quand la politique s'en mêle

\section{Chansons pop : tradapter pour que résonne le sens}

\section{Antoine Guillemain}

\section{(2) OpenEdition}

Journals

Édition électronique

URL : http://journals.openedition.org/traduire/1738

DOI : 10.4000/traduire.1738

ISSN : 2272-9992

\section{Éditeur}

Société française des traducteurs

\section{Édition imprimée}

Date de publication : 20 juin 2019

Pagination : 107-121

ISSN : 0395-773X

\section{Référence électronique}

Antoine Guillemain, «Chansons pop : tradapter pour que résonne le sens », Traduire [En ligne], 240

2019, mis en ligne le 20 juin 2019, consulté le 05 février 2020. URL : http://journals.openedition.org/ traduire/1738; DOI : 10.4000/traduire.1738 


\section{Chansons pop: tradapter pour que résonne le sens}

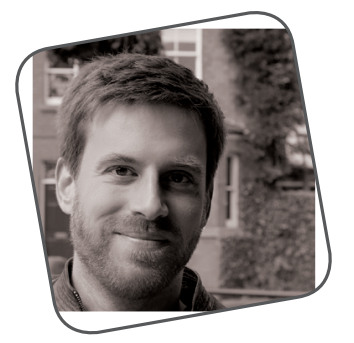

\section{Antoine Guillemain}

Il y a quelques années, j'ai eu l'occasion de constater la popularité de certains sites internet collaboratifs proposant des traductions aux mélomanes non anglophones désireux de comprendre les paroles de la dernière chanson à la mode'.

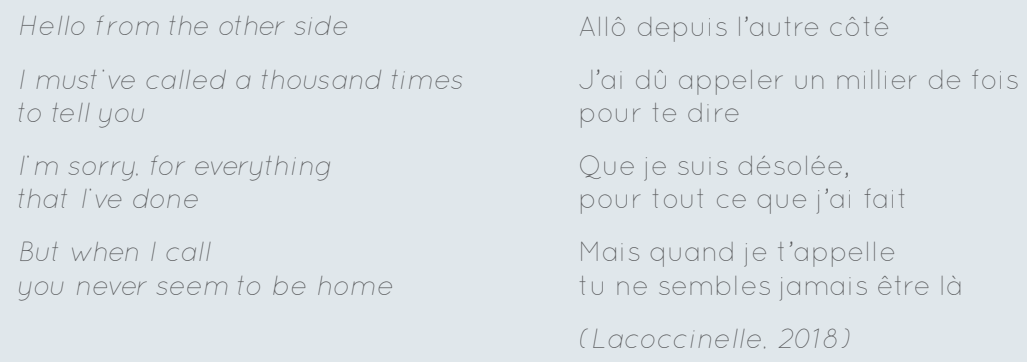

Des traductions au mieux dévitalisées, au pire truffées de tristes contresens. Certes, le but de ces sites n'était pas de produire des textes chantables, mais je ne pouvais m'empêcher de 
penser qu'il y manquait quelque chose d'essentiel pour permettre aux visiteurs d'appréhender pleinement le sens de l'original. Quelque chose qui tenait à la musicalité.

Face à de telles traductions, le grand public dressait un constat sans appel: "Ça sonne quand même nettement moins bien en français.» "C'est intraduisible, on est obligé d'adapter.» Apparemment, il n'existait pour lui que deux solutions: d'un côté, les «traductions», inesthétiques mais recelant la vérité de l'original et, de l'autre, les "adaptations», fort sympathiques mais qui échouaient à traduire véritablement.

En 2014, j'ai résolu d'entreprendre un projet de vulgarisation traductologique dont l'objectif serait double: d'une part, proposer au grand public une alternative aux traductions de chansons discordantes et peu compréhensibles qui circulent sur internet pour lui faire comprendre - lui faire entendre - le sens profond de ses chansons préférées. D'autre part, l'inciter à penser autrement la notion de fidélité en traduction à travers le prisme de la chanson: Iui montrer que la traduction, c'est de l'adaptation, et l'adaptation, de la traduction. Parce que le texte sonne quand la musique est bonne.

\section{Présentation du projet}

Fruit d'un an de travail, leTradapteur est d'abord un site internet ${ }^{2}$ à but non lucratif qui héberge des «tradaptations» françaises de paroles de chansons anglophones à succès: des textes chantables, qui cherchent l'équilibre entre «sens textuel» et «sens musical», prenant en compte à la fois les idées et les paramètres formels - rimes, métrique et rythme, découpe, accentuation et mariage des temps forts musicaux et de l'accent tonique naturel des mots, longueur des voyelles, nature et chantabilité des voyelles et des consonnes, jeux de sons, etc. - des paroles originales. Le site comprend également plusieurs rubriques destinées à vulgariser la traduction de chansons et à fournir un cadre théorique au projet: un glossaire des termes de la tradaptation de chansons, une foire aux questions, un tour d'horizon des tradaptations célèbres, des interviews de tradapteurs, une liste d'outils pratiques (dictionnaires en ligne, bibliographie). Chaque 
tradaptation est assortie d'un commentaire détaillé sur «ce que la chanson veut dire» et d'une série de «notes du tradapteur».

Après avoir tradapté en français une vingtaine de titres - pour la plupart des chansons pop anglophones actuelles ciblant un jeune public -, il m'a semblé indispensable de mettre ces tradaptations en musique pour faire connaitre le site. C'est ainsi que leTradapteur s'est doublé d'une chaîne YouTube ${ }^{3}$, qui héberge à ce jour cina clips, réalisés avec des chanteurs, musiciens et réalisateurs semi-professionnels. Enfin, il m'a paru utile de conférer au projet une dimension didactique. La chaîne diffuse deux Tutos du Tradapteur, vidéos ludiques qui décryptent les paroles de chansons à succès, puis expliquent les enjeux de leur tradaptation à grand renfort d'exemples et de comparaisons.

\section{Fondements théoriques}

La vision de la traduction de chanson en tant que "compromis idéal» entre fond et forme que propose leTradapteur s'inspire de celle défendue par plusieurs théoriciens tels que Peter Low (2005a, 2005b) et Johan Franzon (2008). Low (2005a) identifie cinq critères propres aux chansons auxquels le traducteur doit impérativement prêter attention: chantabilité, sens, naturel, rythme et rime (singability, sense, naturalness, rhythm and rhyme). II introduit le "principe du pentathlon» (Pentathlon Principle), qui suppose «que toute modification [des paroles] ne peut constituer de véritable amélioration que si elle augmente la somme totale des cinq critères, sans quoi elle ne présente aucune réelle valeur ${ }^{4}$ ». Franzon, qui distingue trois "niveaux de chantabilité» (layers of singability), va plus loin en considérant que des paroles véritablement chantables présentent une adéquation "sémantico-réflexive» (semantic-reflexive match) avec la chanson originale et que "la musique [...] ajoute une valeur (sémantique) au contenu des paroles». D'autres théoriciens comme Klaus Kaindl (2005) partagent l'idée que le traducteur doit tenir compte non seulement des paroles originales, mais également de l'ensemble des constituants non verbaux. II considère les paroles d'une chanson comme un "texte multiple»

3. http://youtube.com/leTradapteur

4. Toutes les traductions des citations extraites d'articles en anglais sont de l'auteur du présent article. 
qui "dialogue» non seulement avec la musique, mais aussi avec le clip et les autres chansons du genre. Il déplore:

Même lorsqu'on évoque la dimension non verbale [...], celle-ci est généralement réduite aux contraintes structurelles imposées par la musique au texte verbal. Il est indéniable que la musique influence en amont certains choix traductifs d'ordre syntaxique et prosodique, mais le rôle des constituants non verbaux du texte dépasse largement ses aspects formels et structurels.

Dans cette logique, les tradaptations proposées par leTradapteur ne s'inscrivent pas seulement dans une démarche consistant à réaliser des traductions harmonieuses (agréables à l'oreille) et «dynamiques» (visant à reproduire l'«effet équivalent»que prônait Eugene Nida). Elles cherchent surtout à défendre le caractère essentiellement indivisible du «sens textuel», contenu dans les idées développées et évoquées dans les paroles, et du «sens musical», un sens moins palpable mais tout aussi important, perceptible (1) dans des contraintes formelles évoquées plus haut et (2) dans l'atmosphère infusée par la musique et la performance vocale de l'interprète. Certes, le texte est musique et fond et forme sont imbriqués. Mais il s'agit surtout de dire que le son, c'est du sens, et qu'une traduction de chanson qui ne sonne pas, c'est une traduction qui n'a pas de sens. Elle viendrait rompre cette synergie entre texte et musique qui est l'essence même d'une chanson et équivaudrait à séparer l'inséparable, les paroles de la chanson originale ayant été composées pour épouser parfaitement son arrangement musical (ou l'inverse). À ce sujet, Peter Newmark (2013) observe: "Le partenariat entre les paroles et la musique fait surgir un nouveau sens que ni les paroles seules, ni la musique seule ne sauraient saisir, communiquer ou générer.» II me semble donc que la seule traduction valable pour qui souhaite véritablement appréhender le sens d'une chanson est une tradaptation - un texte chantable -, même si cette traduction n'est fournie que pour éclairer l'original et qu'elle n'est pas destinée à être chantée. En cela, mon opinion diffère quelque peu de celle de Low et Franzon, qui prévoient plusieurs types de traductions selon le skopos du texte.

Le terme "tradaptation» a été utilisé pour la première fois en 1973 par le dramaturge québécois Michel Garneau pour décrire ses propres traductions/adaptations de pièces de Shakespeare, qui ne sont «ni des traductions littérales de 
Shakespeare, ni des adaptations qui modifient largement le fond du texte source. Elles «résistent à toute distinction entre les deux pratiques» (Drouin 2004). Le terme «tradaptation» a été repris notamment en 2004 par Yves Gambier dans un contexte de traduction audiovisuelle:

La pseudo-polarité entre traduction (plus dépendante d'un "original») et adaptation (relative autonomie par rapport à cet "original») ne tient plus: il y a circulation textuelle et surtout synergie entre systèmes sémiotiques. D'où la notion proposée de tradaptation cinématographique (ou transadaptation), apte à englober tous les types de transformations. La tradaptation permet donc [...] de dépasser les oppositions dichotomiques habituelles [...].

Dans son introduction à Translation and Music (2008), Şebnem Susam-Saraeva pense, elle aussi, que s'agissant de "chansons traditionnelles ou populaires, il est souvent impossible - et, selon moi, peu souhaitable - de déterminer où se termine la traduction et où commence l'adaptation». Pour Franzon (2008), "les chansons constituent une profonde remise en question de la tendance à assimiler la traduction à la fidélité au sens». L'indivisibilité des différentes composantes d'une chanson et le brovillage des frontières entre texte et musique me conduisent à broviller également la frontière entre «traduction» et «adaptation» en reprenant à mon compte ce mot-valise. Dans un genre où l'esthétique reflète directement le propos, où le son et la musique contribuent au sens autant que les idées, traduction et adaptation apparaissent plus que jamais comme un continuum.

La multiplicité des contraintes (sémantiques et formelles) et des médiums (texte, musique, clip) permet en outre de concevoir la traduction des paroles comme devant être représentative d'un ensemble plus large. Le «texte» devient une entité plurisémiotique (Kaindl 2005). Dès lors, les idées, les images et leur forme musicale entrent tant en résonance les unes avec les autres et font tant référence à un "contexte» plus vaste qu'elles ne peuvent être appréhendées qu'au regard de la globalité de la chanson. II me semble donc que le tradapteur a tout intérêt à considérer "l'unité de traduction» non plus comme le vers ni la strophe (dont les limites s'avèrent d'ailleurs souvent difficiles à tracer), ni même comme le texte entier, mais comme la chanson dans son ensemble, voire la chanson à l'aune des résonances qu'elle entretient avec ses prédécesseurs. 
Cette approche dialogique et intertextuelle trouve un écho dans les conceptions dites postmodernes de la traduction arguant en faveur de la non-fixité du sens et déconstruisant l'autorité du texte original, qui posent que tout texte contient toujours des traces et des influences d'autres textes ou supports préexistants et entretient avec eux un rapport dépassant largement le cadre du texte lui-même.

S'il envisage la chanson originale comme un carrefour où circulent les sens, le tradapteur se sent d'autant plus fondé à opérer la «dé-surmoi-isation ${ }^{5}$ » qui lui permettra de recréer un texte où résonne l'original.

C'est en effet grâce à un processus créatif que le tradapteur peut

restaurer l'équilibre communicationnel bien souvent rompu par les formes de traduction traditionnelles. Tant qu'on ne [le] traitera pas comme une stratégie légitime, on ne pourra pas en comprendre le bien-fondé ni le rapport qu'[il] entretient avec d'autres formes de traduction plus conventionnelles. (Georges Bastin 2011)

La démarche du projet leTradapteur consiste précisément à traiter la recréation comme une stratégie parfaitement légitime plutôt qu'une solution de rechange, et même comme l'unique moyen de faire émerger le sens de la chanson originale. Le concept de fidélité est ainsi réévalué et devient parfaitement compatible avec la notion de créativité.

Cela ne signifie en aucun cas que le tradapteur ou la tradaptrice fait flèche de tout bois et travaille à sa guise. Les chansons qui ne traduisent que le sens musical de leur original "My Way» de Frank Sinatra, version anglaise de "Comme d'habitude» de Claude François, par exemple) ou celles qui ne retiennent que l'idée-clé et renouvellent entièrement le détail du texte ("Comment te dire adieu» de Françoise Hardy, écrite par Serge Gainsbourg, version française de "It Hurts to Say Goodbye», écrite par Jacob Gold et Arnold Goland, par exemple) ne sont pas des tradaptations, si brillantes soient-elles. Définir la créativité en traduction n'est pas une mince affaire, dans la mesure où ce terme est normalement synonyme de nouveauté. Recréer

5. Préconisée par Jean-René Ladmiral dans une communication faite à l'occasion de la conférence inaugurale du Premier Congrès mondial de traductologie qui s'est tenu à l'Université Paris-Nanterre en avril 2018. 
pour produire une tradaptation consiste justement à exploiter des éléments tangibles de la chanson originale pour façonner de nouvelles idées ou images, absentes de l'original en l'état, mais gravitant dans les mêmes champs lexicaux et reproduisant sa logique interne.

Plus les contraintes sont nombreuses et variées, plus le tradapteur est amené à tirer parti des possibilités de la chanson pour trouver des solutions de traduction. Les théoriciens prônant la créativité en traduction s'accordent à dire que les contraintes du texte source ne sont plus des obstacles, mais des tremplins: elles "permettent et stimulent la créativité de l'acte traductif en plaçant le traducteur dans une situation ou il doit s'efforcer de les dépasser» (Boase-Beier et Holman, in Boase-Beier 2006). Évoquant sa retraduction de l'Enfer dans un entretien (Nigdélian 2016) intitulé "La musique retrouvée de Dante», Danièle Robert commentait: "Plus le texte est "difficile", c'est-à-dire résiste par sa nature même et sa complexité, plus [le traducteur] puise dans sa propre langue tout ce qui peut l'aider à vaincre cette résistance.»

Observons qu'en exploitant les contraintes de l'original, le tradapteur procède de la même manière que les paroliers originaux. Le parolier Michel Arbatz (2011) évoque "l'efficacité que représentent les contraintes imposées» et préconise de les envisager comme «un défi», "un aiguillon à notre imagination». Auteur de nombreuses chansons populaires, Claude Lemesle (2010) abonde en ce sens, citant le philosophe Alain: "Le vrai poète est celui qui trouve l'idée en forgeant le vers. Il faut que la rime soit raison." Ainsi, tradapter une chanson, c'est d'abord retracer «l'impulsion créatrice» (creative impulse, Perteghella et Loffredo, 2006) de l'original. En s'efforçant de faire coïncider le fond et la forme, le tradapteur reproduit - et donc traduit - le processus même de recherche, d'écriture et de création (ibid.) par lequel est passé le parolier original, dont le texte a lui aussi été façonné par la forme et la musique. Une telle traduction, loin d'être une "manipulation gratuite», permet de rétablir l'ordre naturel de production du texte, dont la conception précède la consommation, et de "réveiller le texte source en le sortant de son état de produit fini, fossilisé ». 


\section{Étude de cas}

Pour concrétiser mon propos, je souhaiterais esquisser quelques tendances à partir de ma tradaptation de la chanson "Chandelier» de l'auteure-compositrice-interprète australienne Sia. Le but n'est nullement de dresser l'inventaire exhaustif de toutes les contraintes, stratégies et sources de recréation, mais de donner un bref aperçu de ce qui se jove lorsqu'on tradapte une chanson.

"Chandelier» décrit la spirale dans laquelle s'enferme une femme qui, le soir venu, fait la fête, s'enivre et se livre à des excès pour noyer son mal-être. Ces folles soirées lui permettent de vivre dans l'instant et de se sentir invulnérable, mais la honte l'envahit de nouveau au petit matin. Le clip original met en scène une enfant se livrant à une danse des plus singulières dans un cadre lugubre et dépouillé, qui reflète à l'évidence la détresse du personnage.

\section{Chandelier \\ Party girls don't get hurt \\ Can't feel anything \\ When will I learn? \\ I push it down \\ I push it down}

I'm the one for a good time call

Phone's blowing up

Ringing my doorbell

I feel the love

I feel the love

One two three one two three drink

One two three one two three drink

One two three one two three drink

Throw 'em back till I lose count

I'm gonna swing

From the chandelier

From the chandelier

I'm gonna live

Like tomorrow doesn't exist

Like it doesn't exist

I'm gonna fly

Like a bird through the night

Feel my tears as they dry

I'm gonna swing

From the chandelier

From the chandelier

\section{De délire en délire}

Toute la nuit le coeur en fête

Je suis comme ces filles

Que rien ne blesse

J'ai même plus mal

On même plus mal

Toujours là quand on mappelle

Pour faire la bringue

Brûler la chandelle

Les gens m'aiment

Les gens maiment

Un deux trois et moi je bois

Un deux trois et moi je bois

Un deux trois et moi je bois

Le calice jusqu'à la lie

Moi je vais vivre

De délire en délire

De délire en délire

Moi je vais rire

Comme pour oublier l'avenir

Oublier l'avenir

Moije m'envole

Comme l'oiseau qui s'enflamme

Et qui sèche ses larmes

Moi je vais vivre

De délire en délire

De délire en délire 


\section{Chandelier (suite)}

But I'm holding on for dear life Won't look down won't open my eyes Keep my glass full until morning light 'Cause I'm just holding on for tonight Help me l'm holding on for dear life Won't look down won't open my eyes Keep my glass full until morning light 'Cause I'm just holding on for tonight On for tonight

\section{Sun is up}

I'm a mess

Gotta get out now

Gotta run from this

Here comes the shame here comes the shame

Pre-chorus

\section{Chorus}

And I'm holding on for dear life

Won't look down won't open my eyes Keep my glass full until morning light 'Cause I'm just holding on for tonight Help me I'm holding on for dear life Won't look down won't open my eyes Keep my glass full until morning light 'Cause I'm just holding on for tonight

On for tonight

On for tonight

As I'm just holding on for tonight

On l'm just holding on for tonight

On for tonight

On for tonight

As l'm just holding on for tonight As I'm just holding on for tonight On I'm just holding on for tonight On for tonight

On for tonight

\section{De délire en délire (suite)}

Et moi je me noie dans l'oubli

Et les yeux grands fermés je survis Je me soûle jusqu'au bout de la nuit Accrochée au fil de mes envies Aidez-moi je me noie dans l'oubli Et les yeux grands fermés je survis Je me soûle jusqu'au bout de la nuit Accrochée au fil de mes envies

De mes envies

Il fait jour

Désolée

Je dois partir

Je dois m'enfuir

Je suis pas belle je suis

pas belle

Pré-refrain

Refrain

Et moi je me noie dans l'oubli

Et les yeux grands fermés je survis Je me soûle jusqu'au bout de la nuit Accrochée au fil de mes envies Aidez-moi je me noie dans l'oubli Et les yeux grands fermés je survis Je me soûle jusqu'au bout de la nuit Accrochée au fil de mes envies

De mes envies

De mes envies

Accrochée au fil de mes envies Accrochée au fil de mes envies

De mes envies

De mes envies

Sur un fil jusqu'au bout de la nuit Accrochée au fil de mes envies Accrochée au fil de mes envies De mes envies De mes envies

\section{1) Le tradapteur exploite les contraintes sonores et rythmiques}

Bien souvent, c'est la prosodie qui conditionne la facture du vers. Le vers-clé de cette tradaptation a été dicté par la métrique de la phrase musicale originale, qui se découpe ainsi: «l'm / gonna swing / from the chandelier». Sia fait traîner avec 
force la diphtongue [aI] initiale, à laquelle elle ajoute [m] avant de marquer une pause. En français, il paraît plus naturel de commencer ce vers par "Moi» afin de ne pas séparer le sujet du verbe, c'est-à-dire d'éviter «Je / vais...» au profit de «Moi / je vais...». Notons également la nécessité de composer avec les sons de la langue d'arrivée: la combinaison d'une fricative [3] et de la voyelle semi-fermée [ə] («je») aurait posé un sérieux problème de chantabilité... Et que dire du mot «lustre»pour équivalent de chandelier? J'ai également évité de traduire Like tomorrow doesn't exist par "Comme si demain n'existait pas" (malgré un nombre de syllabes identique), car l'accentuation tonique et musicale (indiquée ici en gras) aurait rendu la phrase française très peu naturelle ("Comme si deux mains n'existaient pas»?). Observons que les mots d'une seule syllabe - même s'ils sont plus rares en français - sont très commodes pour contourner les problèmes de découpe et d'accent tonique. Bien sûr, la performance de l'interprète est cruciale et corrige parfois le tir, lorsqu'elle ne fait pas ressortir davantage ces lourdeurs sonores.

\section{2) Le tradapteur use (et abuse?) de la traduction homophonique (ou «sonotraduction $\left.{ }^{6} »\right)$}

Le vers principal, I'm gonna swing from the chandelier, dont Sia étire la dernière syllabe, musicalement saillante, est particulièrement marquant pour l'auditeur, qui aurait probablement été dérouté de trouver un son différent dans la version française. Le mot "délire» était sans doute ce que le français avait de mieux à offrir pour imiter la sonorité finale de ce vers. De même, on retrouve "J'ai même plus mal» à la place de I push it down, "Brûler la chandelle» à la place de Ringing my doorbell et «Je suis pas belle» à la place de Here comes the shame. Les chansons traduites offrent quantité d'exemples d'imitation des sonorités. Il me semble qu'à l'instar du parolier original, qui ne choisit pas n'importe quel son pour habiller n'importe quelle note, le tradapteur a tendance à reproduire le même son précisément parce que ce son «fait sens». Une tradaptation "sonotraduisante»rend manifeste le fait que «le fond du texte source ne

6. C'est le terme retenu par l'Outranspo, l'«Ouvroir de translation potencial », "groupe de traducteurs, écrivains, chercheurs et musiciens hétéroclite et multilingue, qui se consacre joyeusement aux approches créatives de la traduction». 
réside pas dans ce que dit le texte (le sens) mais dans le médium qu'il utilise pour l'exprimer (le son)» (Jeff Hilson 2013).

\section{3) Le tradapteur compense, déplace et «éclate» les idées}

En optant pour «Moi je vais vivre de délire en délire», j'ai tenté de rendre le sens textuel du vers I'm gonna swing from the chandelier ${ }^{7}$, le terme "délire» pouvant être compris au sens d'«enthousiasme exubérant» (que l'on retrouve dans des expressions telles que «la foule en délire»). La métaphore du plaisir démesuré est par ailleurs éclatée à plusieurs endroits de la chanson (par effet de compensation): on la retrouve dans le deuxième couplet, avec «faire la bringue» et «brûler la chandelle» (comprendre: par les deux bouts), dans le prérefrain avec «je bois le calice jusqu'à la lie». Quid de l'image elle-même (celle de la femme suspendue à son lustre)? On la récupère en partie dans les deuxième et troisième couplets, avec "Accrochée au fil de mes envies» (l'image de la femme qui survit accrochée à un fil servant également à traduire l'm holding on for dear life). Par ailleurs, une évocation de l'image originale figure dans le clip de cette tradaptation: le spectateur y aperçoit à plusieurs reprises un lustre illuminé. On voit ici, je crois, une illustration de la dimension dialogique et intersémiotique du texte-chanson et de la circulation des sens.

\section{4) Le tradapteur fonctionne par associations d'idées et s'appuie sur les clichés, les idiomes, les champs lexicaux et les collocations de sa langue}

Dans «Je me noie dans l'oubli», la notion d'oubli provient de l'image de la femme fermant les yeux (Won't look down won't open my eyes; refus de voir, donc volonté d'oublier). Cependant, ce vers m'a également été inspiré par des expressions telles que «noyer son chagrin dans l'alcool» (en français) et drink oneself into oblivion (en anglais). Notons que les paroliers professionnels opèrent ici encore de la même manière: Claude Lemesle (2010), par exemple, conseille de «savoir décliner toutes les idées annexes

\footnotetext{
7. Précisons que le personnage n'est a priori pas littéralement suspendu à un lustre: ce vers est perçu par certains anglophones comme une simple métaphore de l'excès et du plaisir et, par d'autres, comme une véritable expression figée dénotant la liesse, à rapprocher de swing from the rafters, que j'ai déjà eu l'occasion de traduire par «faire les quatre cents coups».
} 
qui peuvent jaillir de l'idée principale». Michel Arbatz (2011), quant à lui, observe: "Je pense qu'il faut inverser la démarche: partir du matériau-mot et associer les mots par paires ou par séries signifiantes. Le sens vient en second, et vient toujours.» II inventorie différents types d'associations d'idées "par le son, par un cliché, par une image, par proximité de sens, par des enchaînements d'images, par déduction pratique, par le souvenir d'un son, par le souvenir d'une odeur». Bien souvent, une rime cherchée dans le dictionnaire fait finalement surgir une nouvelle idée qui vient se substituer au vers initialement prévu. Dans le refrain, les «larmes» m'auront inspiré «s'enflamme». Par association d'idées avec la métaphore aviaire originale (présente dans l'm gonna fly / Like a bird through the night), ce terme a fait naître l'image du phénix: "Comme l'oiseau qui s'enflamme». "S'enflamme» permet en outre d'évoquer les ardeurs nocturnes du personnage.

\section{5) Le tradapteur puise dans des éléments extérieurs aux paroles}

On touche ici à une dimension beaucoup moins préhensible: le degré auquel la facture générale de la chanson, l'instrumentation musicale, l'interprétation, voire le clip peuvent influencer les choix de traduction, dont le tradapteur lui-même n'est pas forcément conscient. Je ferai ressortir ici deux points. D'abord, la référence au phénix (évoquée plus haut), absente de l'original en l'état, m'a semblé justifiée en raison de la récurrence du prérefrain et du refrain, qui donne l'impression que la femme recommence un cycle et renaît de ses cendres. Ensuite, si le refrain original, avec ses répétitions bancales et ses rimes approximatives, ménage un certain équilibre précaire reflétant l'instabilité du personnage, ce sont l'interprétation torturée de Sia et l'atmosphère pesante du clip qui m’ont permis d'apprécier véritablement la nécessité de conserver des rimes qui se prêtent à une interprétation "plaintive» (c'est-à-dire avec des voyelles fermées plutôt qu'ouvertes), et de ne pas rendre ces rimes trop parfaites.

Observons ici que, dans le cas particulier de la tradaptation des chansons des films d'animation, les visuels à l'écran sont très largement source d'inspiration. Par ailleurs, la synchronisation labiale et le synchronisme cinétique constituent des contraintes supplémentaires. 
En rédigeant ces lignes pour tenter de rationaliser mes choix de traduction, je m'aperçois de nouveau à quel point il est difficile d'attribuer telle trouvaille à telle influence. En écrivant «brûler la chandelle», ai-je été inspiré par le mot chandelier, ai-je voulu recréer la rime de doorbell ou ai-je tenté de trouver une expression idiomatique qui rende le sens textuel de swing from the chandelier? La réponse est probablement: les trois à la fois. Ce travail de catégorisation va sans doute à l'encontre de ce qui se produit lors de la tradaptation d'un texte-chanson, où le tradapteur canalise puis redéploie un réseau d'énergies qui se confondent. L'idéal serait peut-être d'avoir intériorisé la chanson originale au point de pouvoir en traduire les paroles d'une traite sans s'arrêter sur ses constituants individuels?

\section{Perspectives d'avenir}

Si les clips ont reçu un accueil très positif, la dimension traductologique du projet a touché principalement un public de linguistes et de traductologues déjà acquis à la cause. Le grand public, lui, s'attarde surtout sur la voix des chanteurs, leur interprétation, leur jeu ou la réalisation et commente peu les paroles, qui demeurent incontestablement le parent pauvre de la chanson. Lorsqu'il le fait, c'est parfois pour déplorer: "c'est pas les vraies paroles!», "c'est pas la bonne traduction!», croyant voir dans ces tradaptations une imitation fallacieuse. Le grand public semble intimement persuadé que ce que j'ai appelé la "vérité» du sens de l'original ne réside que dans sa littéralité (la notion de traduction littérale étant déjà elle-même toute relative). L'esthétique du texte demeure pour lui un simple ornement, une fioriture, le "packaging» qui habille le message. II reste du chemin à faire pour convaincre de l'indissociabilité du «sens textuel» et du «sens musical» et faire admettre une conception de la traduction comme négociation, où ce qui est perdu ici peut être gagné ailleurs. J'ai également pu constater le désenchantement du public lorsque la performance de l'interprète francophone est trop éloignée de celle de l'interprète original. Pour l'amener à s'intéresser aux paroles (et donc à la démarche traductologique) et lui faire accepter l'idée qu'elles peuvent constituer une traduction valable, il faut manifestement commencer par trouver une voix française capable de reproduire l'original presque à la perfection (comme le font les laboratoires de doublage). C'est 
pourquoi j'envisage aujourd'hui d'autres formats plus courts et plus accessibles, sans clips, qui mettraient l'accent sur le texte et la dimension didactique. De plus, le recours à la traduction homophonique me semble désormais indispensable. En effet, le public du tradapteur de chansons à succès me semble infiniment plus exigeant - et critique - que celui de tout autre traducteur, car il connaît déjà par cœur l'original, dont il a intériorisé les sonorités et avec laquelle il a développé une relation personnelle.

Un projet d'une telle envergure doit être alimenté régulièrement. Je lance ici un appel à collaboration aux paroliers, musiciens et traducteurs professionnels qui sont convaincus, comme Susam-Saraeva (2008), que "le carrefour de la traduction et de la musique peut nous permettre de mieux comprendre ce qu'implique la traduction, jusqu'où ses frontières peuvent être repoussées, et quelles relations elle entretient avec d'autres formes d'expression». À l'heure où le grand public se laisse de plus en plus facilement convaincre que la traduction est automatique, il me semble impératif que nous autres traducteurs nous ouvrions à des médiums et à des formats "grand public». Ce n'est qu'en sortant de notre entre-soi que nous pourrons inciter les non-avertis à changer de grille de lecture sur la traduction. Et faire résonner en eux le sens de ces mots de Marguerite Duras:

Tout le monde sait bien que la traduction n'est pas dans l'exactitude littérale d'un texte, mais peut-être faudrait-il aller plus loin: et dire qu'elle est davantage dans une approche d'ordre musical, rigoureusement personnelle et même, s'il le faut, aberrante. C'est très difficile à dire, c'est un peu ce que je voulais faire, essayer de le dire: les erreurs musicales sont les plus graves. [...] Est-ce qu'il n'y a pas dans la convention du sens respecté une scolarité à retardement qui jove contre la liberté d'un texte, contre sa respiration ou sa folie?

\section{guillemain.antoine@gmail.com letradapteur@letradapteur.fr}

Antoine Guillemain travaille depuis 2012 à la fois comme traducteur littéraire et comme traducteur spécialisé (d'aucuns diront «pragmatique») de l'anglais au français. II a été initié aux métiers de la traduction audiovisuelle au sein de Dubbing Brothers (première société française de doublage) et son mémoire de master s'intéressait à la tradaptation des chansons dans un contexte audiovisuel. Le site internet leTradapteur.fr et la chaîne YouTube associée ont été lancés en octobre 2015. 
ARBATZ Michel, Le Moulin du parolier, Saint Julien Molin Molette, Jean-Pierre Huguet Éditeur, 2011.

BASTIN George, «Adaptation", in BAKER Mona et SALDANHA Gabriela (dir.), Routledge Encyclopedia of Translation Studies, New York (États-Unis), Routledge, 2011, p. 3-6.

BOASE-BEIER Jean, «Loosening the Grip of the Text: Theory as an Aid to Creativity», in PERTEGHELLA Manuela et LOFFREDO Eugenia (dir.), Translation and Creativity: Perspectives on Creative Writing and Translation Studies, Londres, Continuum, 2006, p. 46-56.

DROUIN Jennifer, Macbeth (1978): Michel Garneau, Guelph (Canada), Canadian Adaptations of Shakespeare Project, 2004, www.canadianshakespeares.ca/a_garneau.cfm, consulté le 13 mai 2019.

DURAS Marguerite, «Les Infidélités de L'Amant», in MALROUX Claire (dir.), Actes des quatrièmes Assises de la Traduction littéraire (Arles 1987), Arles (France), Actes Sud, 1988, p. 89-90, www.atlas-citl.org/wp-content/uploads/pdf/4actes.pdf, consulté le 13 mai 2019.

FRANZON Johan, «Choices in Song Translation: Singability in Print, Subtitles and Sung Performance", in The Translator, 2, 2008, p. 373-399.

GAMBIER Yves, «Tradaptation cinématographique», in ORERO Pilar (dir.), Topics in Audiovisual Translation, Amsterdam (Pays-Bas), John Benjamins, 2004, p. 169-181.

HILSON Jeff, «Homophonic Translation: Sense and Sound», in MINORS Helen Julia, (dir.), Music, Text and Translation, Londres, Bloomsbury, 2013, p. 95-105.

KAINDL Klaus, «The Plurisemiotics of Pop Song Translation: Words, Music, Voice and Image», in GORLÉE Dinda L. (dir.), Song and significance: Virtues and Vices of Vocal Translation, Amsterdam (Pays-Bas), Rodopi, 2005, p. 235-262.

Lacoccinelle, Paroles et traduction de la chanson «Hello» par Adele, www.lacoccinelle. net/1042762-adele-hello.html, consulté le 13 mai 2019.

LEMESLE Claude, L'Art d'écrire une chanson, Paris, Eyrolles, 2010.

LOW Peter, «The Pentathlon Approach to Translating Songs», in GORLÉE Dinda L. (dir.), Song and Significance: Virtues and Vices of Vocal Translation, Amsterdam (Pays-Bas), Rodopi, 2005a, p. 185-212.

LOW Peter, «Song Translation », in BROWN K. et al., The Elsevier Encyclopedia of Language and Linguistics, Oxford (Royaume-Uni) Elsevier, 2005b, p. 511-514.

NEWMARK Peter et MINORS Helen Julia, «Art Song in Translation", in MINORS Helen Julia (dir.), Music, Text and Translation, Londres, Bloomsbury, 2013, p. 59-68.

NIGDÉLIAN Valérie, «La musique retrouvée de Dante», in Le Matricule des anges, 173, 2016, p. 44-46.

PERTEGHELLA Manuela et LOFFREDO Eugenia (dir.), Translation and Creativity: Perspectives on Creative Writing and Translation Studies, Londres, Continuum, 2006.

SIA et SHATKIN Jerry, "Chandelier», in 1000 Forms of Fear, Londres, Monkey Puzzle Records, 2014.

SUSAM-SARAEVA Şebnem, «Translation and Music: Changing Perspectives, Frameworks and Significance", in The Translator: Translation and Music, 2, 2008, p. 373-399. 